\title{
Efeitos da intervenção Hatha-Yoga nos níveis de estresse e ansiedade em mulheres mastectomizadas
}

\author{
The effects of hatha yoga exercises on stress and anxiety levels \\ in mastectomized women
}

\author{
Marina Lima Daleprane Bernardi ${ }^{1}$ \\ Maria Helena Costa Amorim ${ }^{1}$ \\ Eliana Zandonade ${ }^{1}$ \\ Danilo Forghieri Santaella ${ }^{2}$ \\ Juliana de Assis Novais Barbosa ${ }^{1}$
}

${ }^{1}$ Universidade Federal do Espírito Santo. Av. Fernando Ferrari 514, Goiabeiras. 29.073-910 Vitória ES Brasil.

mamadaleprane@hotmail.com

${ }^{2}$ Universidade de São

Paulo.
Abstract This article seeks to evaluate the effects of hatha yoga on stress and anxiety levels in mastectomized women. It also investigates the relationship between these levels with the following variables: age; marital status; religion; instruction; profession; smoke addiction; elitism; staging of the disease; and treatment phase. This involved controlled random clinical trial sampling of 45 mastectomized women treated at the Ilza Bianco outpatient service of Santa Rita de Cássia Hospital in the Brazilian state of Espirito Santo from March to November 2010. The experimental group participated in 6 individually-applied sessions with incentive for ongoing home practice and was reevaluated after the period, whereas the control group was re-evaluated after a proportional peri$o d$. For the study of the variables, the interview and recording on a form technique was used, along with the Anxiety Trait and State Test, and the Stress Symptoms and Signs Test. For statistical treatment, the Statistical Pack for Social Sciences was used. The data are statistically significant and have shown that hatha yoga exercises decrease stress and anxiety in the experimental group. No connection between confounding variables and anxiety and stress levels was found.

Key words Breast neoplasms, Mastectomy, Anxiety, Emotional and physical burnout, Yoga
Resumo O objetivo deste artigo é avaliar os efeitos da Hatha-Yoga nos niveis de estresse e ansiedade de mulheres mastectomizadas, bem como examinar a relação destes níveis com as variáveis: idade, estado civil, religião, escolaridade, profissão, tabagismo, etilismo, estadiamento da doença e fase de tratamento. Trata-se de um ensaio clínico aleatorizado controlado cuja amostra foi constituída por 45 mulheres mastectomizadas atendidas no Ambulatório Ilza Bianco do Hospital Santa Rita de Cássia, Espírito Santo, Brasil, de março a novembro de 2010. O grupo experimental participou de 6 intervenções aplicadas individualmente com incentivo para a prática domiciliar e foi reavaliado após esse período, enquanto o grupo controle foi reavaliado em período proporcional. Para o estudo das variáveis, foi utilizada a técnica de entrevista com registro em formulário, os Inventários de Ansiedade Traço e Estado e o Sinais e Sintomas de Stress. Para o tratamento estatístico foi utilizado o Pacote Estatístico para Ciências Sociais. Os dados encontrados são estatisticamente significantes e demonstraram que a intervenção Hatha-Yoga diminuiu o estresse e a ansiedade no grupo experimental. Não foi encontrada relação das variáveis de confundimento com os níveis de ansiedade e estresse.

Palavras-chave Neoplasias da mama, Mastectomia, Ansiedade, Exaustão emocional e física, Ioga 


\section{Introdução}

O câncer de mama é o segundo tipo mais frequente no mundo e o mais comum na população feminina, crescendo $22 \%$ a cada ano dentre os casos novos em mulheres. Apesar de ter um bom prognóstico, as taxas dessa doença permanecem altas, provavelmente pelo diagnóstico tardio ${ }^{1}$. Desse modo, o câncer de mama é o tipo de neoplasia mais temida por essa população, seja pela sua alta frequência e mortalidade, seja pelos efeitos psicológicos nas portadoras. Tais efeitos envolvem negativamente a percepção da sexualidade e da própria imagem pessoal mais do que em qualquer outro tipo de câncer².

As mulheres que se submetem ao tratamento de câncer de mama passam por grande sofrimento, visto que os procedimentos em questão são agressivos, invasivos e até mesmo mutilantes. A cirurgia de remoção da massa tumoral é um procedimento provável para a maioria das mulheres que apresentam câncer de mama e que causa impacto negativo em sua qualidade de vida $^{3,4}$. As mulheres mastectomizadas apresentam comprometimento em sua imagem corporal, o que envolve suas emoções, pensamentos, a imagem que tem de si mesma e de sua sexualidade, e o modo de se relacionarem com os outros ${ }^{3}$.

O estado de tensão gerado pelo estresse, de um modo geral, é determinado conforme a capacidade do indivíduo em adaptar-se física, mental e socialmente às exigências impostas por esses estímulos nas mudanças ocorridas em sua vida, sejam estas positivas ou negativas 5 . Porém, o estado de tensão decorrente de toda essa situação vivida pelas mulheres mastectomizadas é referido por muitas pacientes como fator de enfraquecimento ao combate da doença e favorecedor tanto de sua recorrência, quanto de sua progressão ${ }^{6}$.

Diante dessa situação, Silva e Santos ${ }^{7}$ enfatizam que os cuidados às mulheres acometidas pelo câncer de mama não se esgotam em procedimentos cirúrgicos, quimio e radioterápicos, mas devem ser permanentes. Para isso, faz-se necessário um processo de recuperação física e psicológica dessa mulher que envolva o bem-estar psicossocial e espiritual a que todos os indivíduos têm direito. A recuperação teria como objetivo tratar ou atenuar as incapacidades causadas pela doença ou pelos efeitos colaterais do tratamento com o objetivo de promover sua reintegração social e qualidade de vida. Santos e Viei$\mathrm{ra}^{3}$ e Amorim ${ }^{8}$ reforçam essa situação quando afirmam a necessidade de intervenções que ofereçam a mulher com diagnóstico de câncer de mama possibilidades de trabalhar o seu corpo com o mundo e com o outro.

Sob a perspectiva de cuidados integrais e promoção de saúde, observa-se um crescente interesse pela prática de Yoga como disciplina que considera o indivíduo sob uma perspectiva abrangente, sob os níveis físico, mental, espiritual e social'. Nesse contexto a prática de Yoga tem sido bem difundida no Ocidente e considerada benéfica à saúde, seja na melhoria do potencial de saúde dos indivíduos sãos, na cura de doenças, ou no gerenciamento do estresse ${ }^{10}$. Pashard ${ }^{11}$ em uma investigação sobre os efeitos do Yoga no organismo do indivíduo afirma que essa disseminação da prática não tem a pretensão de substituir a conduta médica tradicional, mas de sua integração na assistência a saúde, favorecendo o bem-estar dos indivíduos.

O Hatha-Yoga é uma vertente do Yoga que busca desenvolver o potencial do corpo estabelecendo sua integração com a mente, atenuando, desta forma, problemas físicos e emocionais. Trata-se da ramificação do Yoga mais difundida no Ocidente, cujo sistema é composto por posturas corporais, controle da respiração, inibição sensorial, concentração e meditação, além de diversas técnicas de limpeza orgânica ${ }^{12}$. Sua prática é capaz de produzir efeitos revigorantes propiciando ao praticante um estado permanente de energia, serenidade, autoconfiança, equilíbrio físico e emocional, clareza mental e resistência à fadiga, substituindo ansiedades, fobias, conflitos e comportamentos neuróticos. Para alcançar essas sensações é necessário perceber cada momento da experiência, sem forçar o corpo além do limite confortável ${ }^{13}$. No entanto, apesar do grande potencial terapêutico e de promoção da saúde nos indivíduos, esta prática pode não alcançar o mesmo efeito positivo para todos os praticantes, como recomendado pelas tradições indianas e por muitas pesquisas científicas ${ }^{14}$.

Partindo desse princípio, estudos têm examinado a influência da prática de Yoga no relaxamento, no bem-estar, no enfrentamento, na aceitação e na saúde física de pessoas com câncer, sendo o de mama a neoplasia mais estudada ${ }^{15}$. A utilização do Hatha-Yoga como prática auxiliar no tratamento do câncer de mama demonstra que só tem a somar na reabilitação das portadoras, e por isso o potencial dessa intervenção deve ser mais bem explorado em pesquisas nessa área ${ }^{16}$. Buettner et al. ${ }^{17}$ constatam essa afirmação em um estudo transversal revelando as formas de terapia complementar e alternativa utilizadas por 2.332 mulheres sobreviventes de câncer de 
mama. O Yoga, apesar de não ser terapia mais procurada entre as mulheres, proporcionou melhor qualidade de vida entre elas. Assim, em consideração à crescente utilização dessa intervenção no campo científico é importante mencionar para melhor esclarecimento, que diversos autores utilizam a intervenção Hatha-Yoga em seus estudos clínicos, descrevendo-a como uma modalidade da mesma ou como apenas Yoga.

Diante das evidências científicas a respeito do Hatha-Yoga como intervenção para mulheres mastectomizadas, e diante da escassez de estudos nacionais acerca dos benefícios obtidos através de sua prática, algumas questões emergiram, constituindo-se como motivação para realização deste estudo entre as quais: a intervenção Hatha-Yoga pode reduzir o estado de ansiedade e os sinais e sintomas de estresse em mulheres mastectomizadas? As variáveis como idade, estado civil, religião, escolaridade, profissão, tabagismo, etilismo, estadiamento da doença, e fase de tratamento poderiam ter alguma influência nessas medidas?

\section{Objetivos}

- Avaliar os efeitos da intervenção Hatha-Yoga na ansiedade, nos sinais e sintomas de estresse em mulheres mastectomizadas;

- Examinar a relação dos níveis de ansiedade e dos sinais e sintomas de estresse com as variáveis de confundimento: idade, estado civil, religião, escolaridade, profissão, tabagismo, etilismo, estadiamento da doença e fase do tratamento durante a participação dessas mulheres a fim de verificar a comparabilidade das amostras.

\section{Método}

Trata-se de um ensaio clínico aleatorizado. Participaram do estudo 45 mulheres mastectomizadas atendidas no Ambulatório Ylza Bianco localizado no Hospital Santa Rita de Cássia (HSRC), criado e mantido pela Associação Feminina de Ensino e Combate ao Câncer (Afecc). A amostra foi constituída por 44 mulheres, sendo 19 no grupo controle e 26 no grupo experimental.

A constituição da amostra se fez pela demanda de mulheres encaminhadas ao Programa de Reabilitação para Mulheres Mastectomizadas (Premma) por intermédio de profissionais que prestam assistência às portadoras de câncer de mama na instituição HSRC. Os grupos controle e experimental foram constituídos aleatoriamente, através de sorteio, no primeiro contato com as voluntárias à medida que estas concordaram em participar do estudo e manifestaram a aquiescência, com a assinatura do Termo de Consentimento, documento preenchido em três vias, uma para o prontuário, uma para a cliente e outra para a pesquisadora.

Deste modo, foram incluídas do estudo mulheres acima de 21 anos, mastectomizadas em diferentes etapas de tratamento e sem qualquer contato prévio com o Programa de Reabilitação para Mulheres Mastectomizadas (Premma). Foram adotados como critérios de exclusão a presença de: metástase à distância e de recidiva da doença, qualquer tipo de psicose aparente, deficiência mental, quadro de demência, déficit de audição e/ou linguagem que pudessem comprometer a entrevista ou a intervenção.

Após a aleatorização entre os grupos, as mulheres do grupo experimental foram convidadas a participarem da intervenção Hatha-Yoga. A intervenção foi aplicada individualmente nas voluntárias, em local calmo, sob o assoalho, com a utilização de colchonetes e travesseiros como material de apoio. A intervenção foi composta por seis práticas de Hatha-Yoga com duração de 45 minutos, cujo roteiro é baseado em um protocolo composto por um momento de acolhimento e interiorização das mulheres voluntárias, conscientização da respiração diafragmática, realização de posturas corporais, realização de exercícios respiratórios, relaxamento, exercícios de concentração preparatórios para meditação. Além disso, essas mulheres receberam um guia para prática de Hatha-Yoga e foram incentivadas a praticarem a intervenção em seus domicílios.

As intervenções Hatha-Yoga foram monitoradas com as medidas de pressão arterial, frequência cardíaca e respiratória e a sensação referida pela mulher após cada prática. Após o encerramento de cada intervenção foi reservado para as participantes da intervenção um momento para esclarecimento de dúvidas e depoimentos, bem como para a instrutora reforçar a necessidade da prática domiciliar regular utilizando um guia de orientação.

A análise dos efeitos da intervenção HathaYoga nos níveis de ansiedade e estresse das mulheres mastectomizadas foi controlada mediante as variáveis: idade, situação conjugal, grau de instrução, profissão, religião, tabagismo, etilismo, estadiamento da doença, etapa do tratamento (Figura 1). Tais variáveis foram coletadas por meio de um formulário, com exceção da variável 


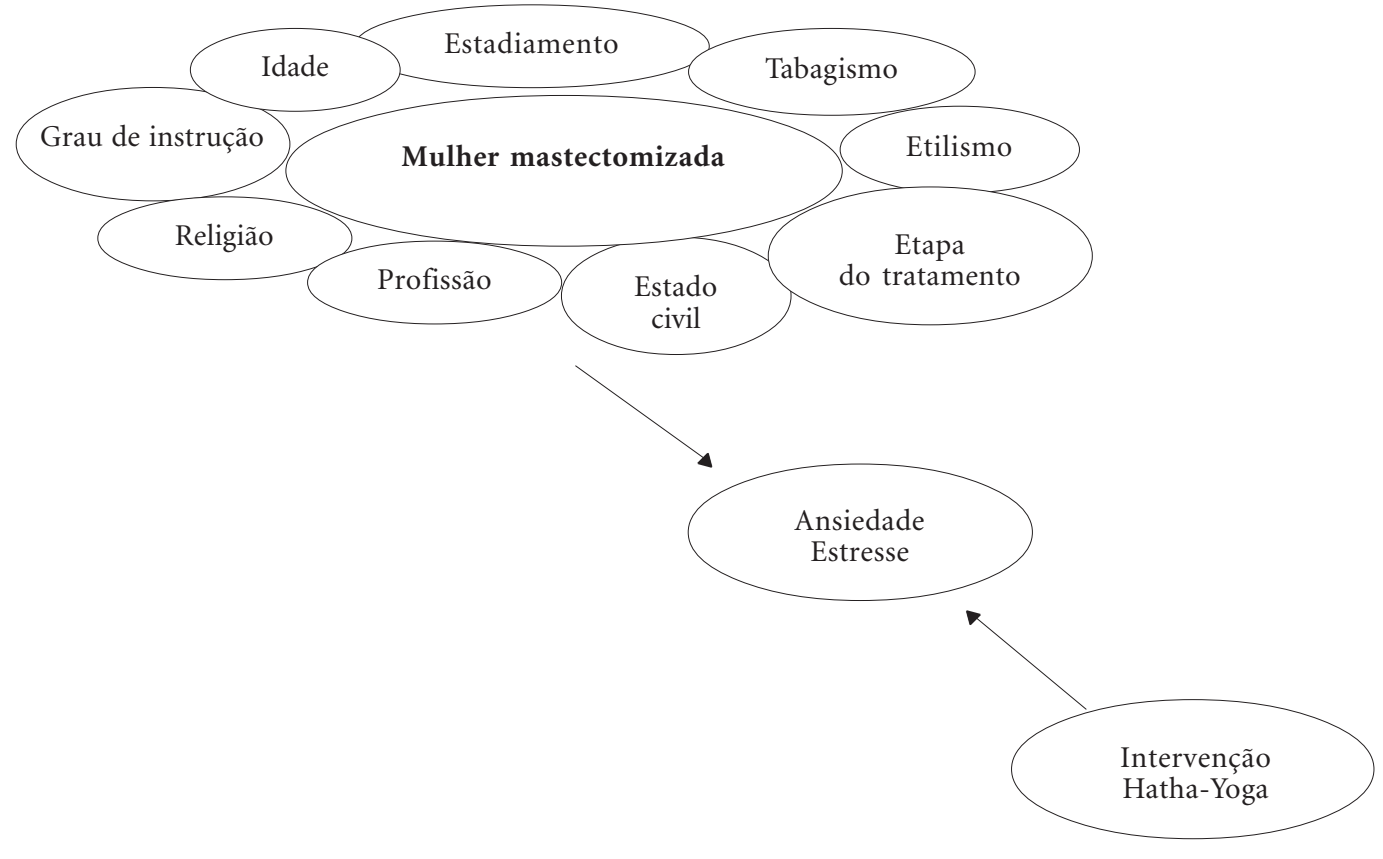

Figura 1. Relação entre as variáveis - Vitória, 2010

estadiamento que foi coletada através do prontuário da mulher e inserida ao formulário.

O traço e estado de ansiedade foram avaliados por meio do instrumento STAI- STATE TRAIT ANXIETY INVENTORY, elaborado por Spielberger et al. ${ }^{18}$, reconhecido no Brasil como Inventário de Ansiedade (A-Traço) e Estado (BEstado) ou IDATE, validada em $1979^{19}$. Esse instrumento é composto por 20 itens dedicados à avaliação do traço de ansiedade característico da mulher e 20 à avaliação de seu estado de ansiedade no momento que constatou a doença. A frequência do traço de ansiedade é analisada dentro das opções: quase nunca (1), às vezes (2), frequentemente (3), quase sempre (4); enquanto no estado de ansiedade estão disponíveis as opções: não (1); um pouco (2), bastante (3), totalmente (4). A pontuação desses itens varia entre 20 e 80 pontos, podendo indicar níveis de ansiedade baixo (20 a 40), médio (40 a 60) e alto (60 a 80).

A avaliação dos sinais e sintomas de estresse foi avaliada pelo instrumento Lista de Sinais e Sintomas de Estresse (LSS/VAS) desenvolvido por Vasconcelos ${ }^{20}$. O instrumento é composto por 59 questões relativas aos sintomas fisiológicos, emocionais, cognitivos e sociais de estresse. A volun- tária assinala a frequência com que ocorrem os sintomas que sente em uma escala de (0) nunca, (1) raramente, (2) frequentemente, e (3) sempre. Para classificação dos níveis de estresse é tomada como referência a pontuação mínima como 10 e a máxima como 177, considerando os níveis de estresse como baixo (10 a 66 pontos), médio (67 a 122 pontos) e alto (123 a 177 pontos).

Ao entrar em contato com a pesquisadora as mulheres foram devidamente orientadas, conforme o enquadramento ao qual foram agrupadas, quanto aos objetivos da pesquisa e ao procedimento ao qual serão submetidas. Nesse primeiro momento as mulheres foram entrevistadas para coleta das variáveis de controle, para aplicação dos questionários para avaliação do traço de ansiedade, estado de ansiedade e sinais e sintomas de estresse.

A partir dessa entrevista o grupo experimental foi submetido a seis intervenções Hatha-Yoga, cerca de duas semanas, sendo novamente avaliado em seu estado de ansiedade e sinais e sintomas de estresse após a última intervenção. O grupo controle apenas aguardou o período correspondente as intervenções Hatha-Yoga, sendo novamente avaliado como o grupo experimen- 
tal. A Figura 2 ilustra o panorama geral do procedimento para a coleta dos dados.

Ao final de cada prática as voluntárias relataram suas respectivas sensações, que foram registradas pela pesquisadora. Esta ainda descreveu quando necessário, em anexo a esses dados, as possíveis limitações em realizar os exercícios, bem como as devidas modificações realizadas para a execução do mesmo.

Para análise estatística dos dados foi utilizado o Pacote Estatístico para Ciências Sociais (SPSS) - versão 17.0 e fixado um nível de significância de $5 \%$, correspondendo a $\mathrm{p}=0,05$ (limite de confiança de 95\%). Foram feitas associações entre variáveis qualitativas através do teste qui-quadrado, e comparação das médias de idade através do teste t. Este teste também foi utilizado nas comparações entre o momento inicial e o final.

Esta pesquisa encontra-se em consonância com as determinações éticas previstas na Resolução no 196/96²1 da Comissão Nacional de Ética em Pesquisa (Conep) e foi aprovada pelo Comitê de Ética em Pesquisa da Universidade Federal do Espírito Santo (UFES).

\section{Resultados}

Este estudo teve como objetivo avaliar os efeitos da intervenção Hatha-Yoga nos níveis de ansiedade e estresse de mulheres mastectomizadas, bem como, examinar a relação desses níveis com as variáveis: idade, estado civil, religião, escolaridade, profissão, tabagismo, etilismo e estadia-

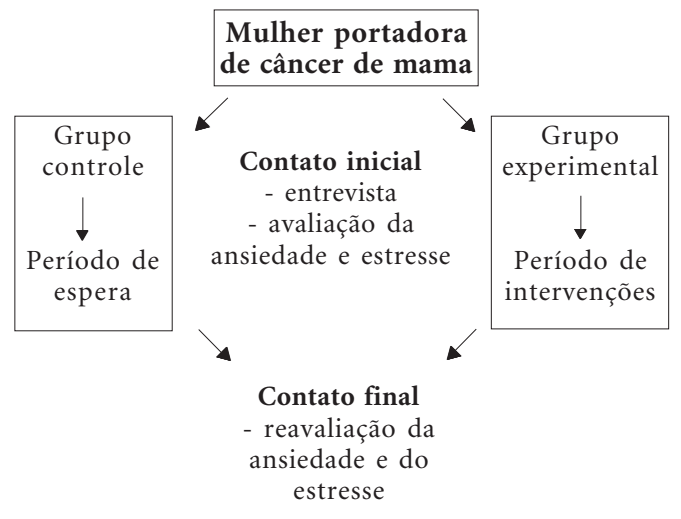

Figura 2. Procedimento para coleta de dados. Vitória, 2010. mento da doença, e fase de tratamento dessas mulheres.

A Tabela 1 apresenta a caracterização da amostra através dos valores absolutos e os percentuais das variáveis qualitativas nos grupos controle e experimental, e demonstra que não houve diferença significativa entre o grupo controle e o experimental em relação a essas variáveis, pois os valores de p são $>0,05$. Deste modo pode-se afirmar que há homogeneidade entre os grupos experimental e controle, e estes são, portanto, comparáveis.

Mais de $60 \%$ das mulheres são casadas, no grupo controle $(68,4 \%)$ e no grupo experimental $(65,4 \%)$. As religiões católica e evangélica foram predominantes em ambos grupos, no experimental essa porcentagem foi de 42 e $34 \%$, e no controle 36 e 58\%, respectivamente. Nos dois grupos a amostra apresenta distribuição equilibrada quanto ao grau de instrução. No grupo experimental $57,9 \%$ das mulheres relatam ser desde analfabetas até ter o $2^{\circ}$ grau incompleto e $42,2 \%$ relatam ter do $2^{\circ}$ grau completo ao o ensino superior completo, enquanto no grupo controle esses valores estão mais equilibrados sendo $52,8 \%$ e $47,4 \%$, respectivamente. Quanto à profissão, 66,2\% dessas mulheres estão inseridas no mercado de trabalho, como autônomas (31,6\% no grupo controle e 34,6 no experimental) ou com vínculo empregatício (31,6\% no grupo controle e 34,6 no experimental). Atualmente, a maioria delas não faz uso de tabaco e de álcool, incluindo as ex-tabagistas e ex-alcoolistas, nos grupos experimental (92,3\% nas duas variáveis) e controle (100\% nas duas variáveis). O estadiamento no grupo experimental mais significativo foi o estadiamento II (40,3\%) enquanto no grupo controle os estadiamentos II e II foram predominantes e equivalentes $(36,8 \%)$. Nos dois grupos houve um equilíbrio de mulheres em fase pós-cirúrgica (36,8\% no grupo controle e $26,9 \%$ no experimental), quimioterapêutica $(31,6 \%$ no grupo controle e $23,1 \%$ no experimental) e radioterapêutica $(21,1 \%$ no grupo controle e $46,1 \%$ no experimental), sendo pouco significante o percentual de mulheres em hormonioterapia.

Não há diferenças estatisticamente significativas ( $\mathrm{p}=0,09)$ entre as médias de idade dos grupos controle $(49,0)$ e experimental $(53,0)$.

A maior parte das mulheres nos grupos controle e experimental apresentam traço com grau de média ansiedade. Comparando a frequência do traço de ansiedade entre os grupos observase que as médias estão muito próximas e, portanto, não há diferença estatística significante 
Tabela 1. Valores absolutos e percentuais da distribuição da amostra de mulheres mastectomizadas nos grupos controle e experimental das variáveis controladas. HSRC/Afecc. Vitória/ES, março - novembro/ 2010 .

\begin{tabular}{|c|c|c|c|c|c|c|}
\hline \multirow{3}{*}{ Variável } & \multirow{3}{*}{ Categoria } & \multicolumn{4}{|c|}{ Grupo } & \multirow[b]{3}{*}{ p-valor } \\
\hline & & \multicolumn{2}{|c|}{ Controle } & \multicolumn{2}{|c|}{ Experimental } & \\
\hline & & $\mathrm{n}$ & $\%$ & $\mathbf{n}$ & $\%$ & \\
\hline \multirow[t]{4}{*}{ Estado civil } & Solteira & 3 & 15,8 & 4 & 15,4 & 0,623 \\
\hline & Casada / vive como casada & 13 & 68,4 & 17 & 65,4 & \\
\hline & Divorciada / Separada & 1 & 5,3 & 4 & 15,4 & \\
\hline & Viúva & 2 & 10,5 & 1 & 3,8 & \\
\hline \multirow[t]{6}{*}{ Religião } & Católica & 7 & 36,8 & 11 & 42,3 & 0,379 \\
\hline & Protestante & 10 & 52,6 & 9 & 34,6 & \\
\hline & Espírita & - & - & 1 & 3,8 & \\
\hline & Duas ou mais & - & - & 3 & 11,5 & \\
\hline & Sem religião, mas espiritualizado & - & - & 1 & 3,8 & \\
\hline & Outras & 2 & 10,5 & 1 & 3,8 & \\
\hline \multirow[t]{7}{*}{ Grau de Instrução } & Analfabeto & 1 & 5,3 & 2 & 7,7 & 0,844 \\
\hline & $1^{\circ}$ grau incompleto & 4 & 21,1 & 9 & 34,6 & \\
\hline & $1^{\circ}$ grau completo & 4 & 21,1 & 3 & 11,5 & \\
\hline & $2^{\circ}$ grau incompleto & 1 & 5,3 & 2 & 7,7 & \\
\hline & $2^{\circ}$ grau completo & 6 & 31,6 & 6 & 23,1 & \\
\hline & $3^{\circ}$ grau incompleto & 0 &, 0 & 1 & 3,8 & \\
\hline & $3^{\circ}$ grau completo & 3 & 15,8 & 3 & 11,5 & \\
\hline \multirow[t]{4}{*}{ Profissão } & Aposentada & - & - & 2 & 7,7 & 0,524 \\
\hline & Autônoma & 6 & 31,6 & 9 & 34,6 & \\
\hline & Dona de casa & 7 & 36,8 & 6 & 23,1 & \\
\hline & Empregada & 6 & 31,6 & 9 & 34,6 & \\
\hline \multirow[t]{3}{*}{ Tabagismo } & Sim & - & - & 2 & 7,7 & 0,196 \\
\hline & Não & 16 & 84,2 & 16 & 61,5 & \\
\hline & Ex-fumante & 3 & 15,8 & 8 & 30,8 & \\
\hline \multirow[t]{3}{*}{ Alcoolismo } & Sim & 0 & 0,0 & 2 & 7,7 & 0,444 \\
\hline & Não & 15 & 78,9 & 18 & 69,2 & \\
\hline & Já bebi, mas parei & 4 & 21,1 & 6 & 23,1 & \\
\hline \multirow[t]{5}{*}{ Estadiamento } & 0 & 0 & 0,0 & 1 & 3,8 & 0,323 \\
\hline & I & 3 & 15,8 & 7 & 26,9 & \\
\hline & II & 7 & 36,8 & 11 & 40,3 & \\
\hline & III & 7 & 36,8 & 4 & 15,4 & \\
\hline & Sem informação & 2 & 10,5 & 2 & 7,7 & \\
\hline \multirow[t]{6}{*}{ Etapa do tratamento } & Cirurgia & 7 & 36,8 & 7 & 26,9 & 0,295 \\
\hline & Cirurgia e quimioterapia & 7 & 36,8 & 6 & 23,1 & \\
\hline & Cirurgia e radioterapia & 0 & 0,0 & 5 & 19,2 & \\
\hline & Cirurgia, quimio e radioterapia & 4 & 21,1 & 7 & 26,9 & \\
\hline & Cirurgia, radio, quimio e hormonioterapia & 1 & 5,3 & 1 & 3,8 & \\
\hline & Total & 19 & 100,0 & 26 & 100,0 & - \\
\hline
\end{tabular}

Fuente: Elaboración propia a partir de datos del Sistema Nacional de Información en Salud (SINAIS), el Instituto Nacional de Estadística, Geografía e Informática (INEGI) y el Consejo Nacional de Población (CONAPO)

entre os mesmos, sendo $\mathrm{p}=0,793$ como demonstra a Figura 3.

A Figura 4 mostra que as mulheres do grupo controle e do experimental apresentam estado de média ansiedade no momento inicial. Neste momento os grupos não apresentam diferença estatisticamente significante entre as médias, o que demonstra homogeneidade da amostra, sendo $\mathrm{p}=0,096$.

Ainda na Figura 4, observa-se que do momento inicial para o final não houve diferença significativa no grupo controle, permanecendo com média ansiedade, sendo $\mathrm{p}=0,866$. Entretanto, o grupo experimental diminuiu o estado 
de ansiedade de forma significativa $(p=0,000)$, passando ao estado de baixa ansiedade. Além disso, houve uma diferença estatisticamente significante entre os grupos controle e experimental no momento final, onde $\mathrm{p}=0,000$.

Perfil de médias - barras verticais denotam IC de $95 \%$

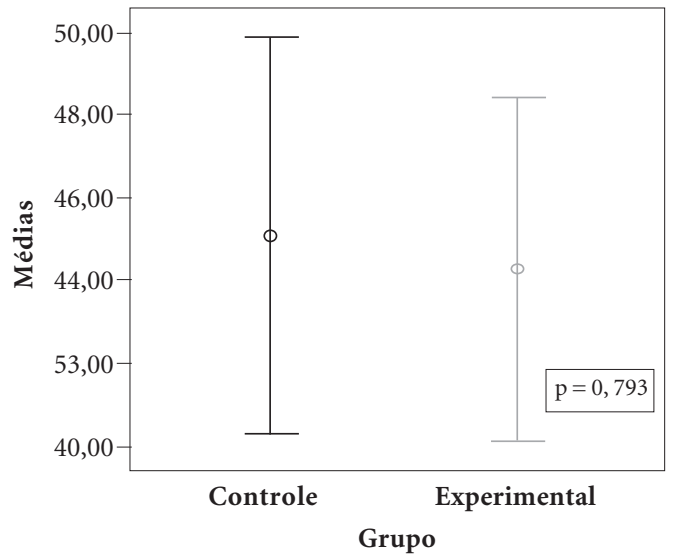

$\begin{array}{cc}\text { Controle: } & \text { Experimental: } \\ \text { Média }=45,11 & \text { Média }=44,31 \\ \text { Mediana }=44,0 & \text { Mediana }=44,5 \\ \text { Desvio Padrão }=9,93 & \text { Desvio padrão }=10,15\end{array}$

Figura 3. Distribuição da amostra de mulheres mastectomizadas quanto ao traço de ansiedade do grupo controle e experimental. HSRC/ Afecc. Vitória/ES, março - novembro/2010.
A Figura 5 demonstra que as mulheres do grupo controle e do experimental apresentam baixos níveis de sinais e sintomas de estresse no momento inicial. Neste momento os grupos não apresentam diferença estatisticamente significante entre as médias, o que demonstra homogeneidade da amostra, sendo $p=0,465$. Observa-se que do momento inicial para o final não houve diferença significativa no grupo controle, sendo $\mathrm{p}=$ 0,177 . Entretanto, o grupo experimental diminuiu de forma significativa seus sinais e sintomas de estresse, passando a níveis de ansiedade ainda mais baixos $(p=0,000)$. Ao final houve diferença estatisticamente significante entre os grupos controle e experimental, onde $\mathrm{p}=0,233$.

\section{Discussão}

No que diz respeito à relação das variáveis: idade, estado civil, religião, escolaridade, profissão, tabagismo, etilismo, estadiamento da doença e etapa do tratamento com os níveis de ansiedade e estresse, não houve associação significativa entre as variáveis $(\mathrm{p}>0,05)$ e, por isso, não houve influencia dessas variáveis de confundimento nas dependentes.

Constatou-se que a maior parte das mulheres nos grupos controle e experimental apresentaram traço e estado de ansiedade no momento inicial com grau de média ansiedade, ou seja, além de apresentarem médios níveis de ansiedade em
Perfil de médias - barras verticais denotam IC de 95\%

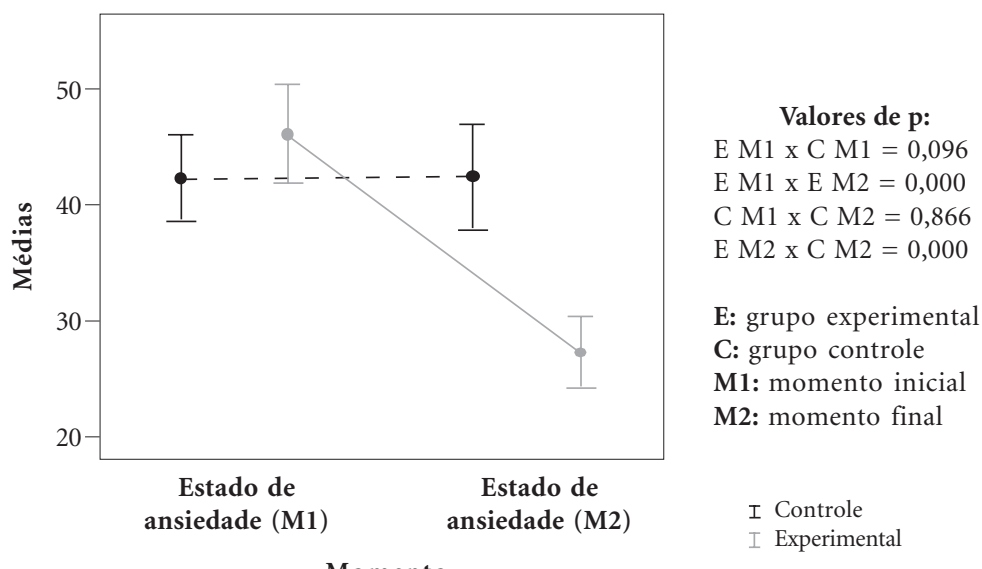

Figura 4. Distribuição de acordo quanto aos níveis de estado de ansiedade nos momentos inicial (M1) e final (M2) nas mulheres mastectomizadas do grupo controle e experimental. HSRC/Afecc. Vitória/ES, março - novembro/2010. 
Perfil de médias - barras verticais denotam IC de 95\%

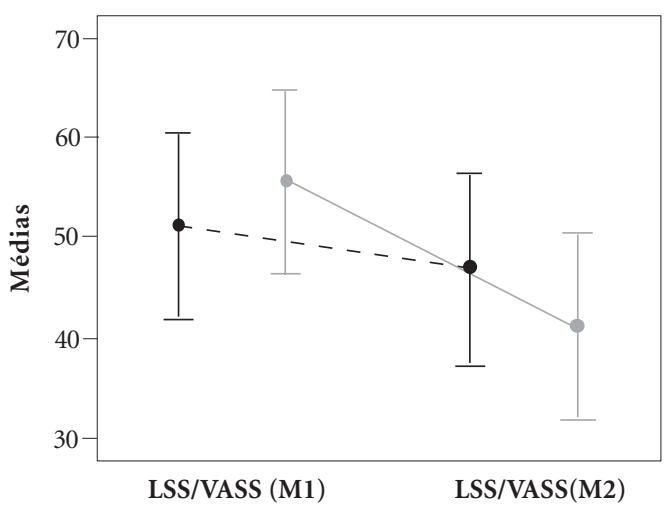

Valores de p:

$\mathrm{E} M 1 \times \mathrm{C} M 1=0,465$

$\mathrm{E} M 1 \times \mathrm{E} \mathrm{M} 2=0,000$

$\mathrm{C} \mathrm{M} 1 \times \mathrm{C} \mathrm{M} 2=0,177$

$\mathrm{E} M 1 \times \mathrm{C} M 1=0,233$

E: grupo experimental

C: grupo controle

M1: momento inicial

M2: momento final

I Controle

I Experimental

Figura 5. Distribuição de acordo quanto aos níveis de sintomas de estresse nos momentos inicial (M1) e final (M2) nas mulheres mastectomizadas do grupo controle e experimental. HSRC/Afecc. Vitória/ES, março - novembro/2010.

seu cotidiano elas apresentavam níveis de média ansiedade no momento da entrevista. Diante dessa situação questionou-se até que ponto o diagnóstico de câncer de mama, bem como o tratamento leva ao aumento nos níveis de ansiedade se essas mulheres são normalmente ansiosas em grau médio.

Paraguassu e Nogueira ${ }^{22}$ relacionaram o estresse com a variável traço de ansiedade em pacientes mastectomizadas frequentadoras de um programa de reabilitação e encontraram significância estatística, resultado da influência da história e do modo de vida da mulher e de sua personalidade. No entanto, apesar de no presente estudo as voluntárias apresentarem baixos níveis de estresse inicialmente, a análise das autoras possibilita um entendimento que justifique o traço e o estado de ansiedade média nessas mulheres.

A diminuição do estado de ansiedade e dos sinais e sintomas de estresse após o período de intervenção estão em concordância com esses resultados, um estudo comparativo constatou a diminuição do estado e traço de ansiedade, além de uma correlação positiva entre estes com os sintomas de estresse durante 6 semanas de intervenção Yoga quando comparada à terapia de suporte educativa oferecida pelo hospital, em mulheres com recente diagnóstico de câncer de mama em grau II e III que realizavam o tratamento convencional em um centro de câncer ${ }^{23}$. Estudo não controlado constatou em 8 práticas de Yoga me- lhoria na qualidade de vida de mulheres com câncer de mama que receberam a fase I e II da quimioterapia, além de uma significativa diminuição no estado e traço de ansiedade e de maiores níveis de satisfação no período de 1 mês ${ }^{24}$.

A partir da década de 90, os estudos clínicos acerca dos efeitos do Yoga ou Hatha-Yoga em diferentes indivíduos, passaram a ter mais destaque inclusive no que diz respeito ao câncer. Deste modo, são encontrados benefícios diversos provenientes da prática de Hatha-Yoga às pacientes portadoras de câncer de mama em diversas etapas do tratamento. De forma geral, tais benefícios estão relacionados à melhora nos níveis de estresse ${ }^{6,16,23,25}$, ansiedade $^{6,23,25}$, depressão $0^{6,25}$, humor ${ }^{16,26,27}$, qualidade de vida ${ }^{16,17,25,27,28}$, fadiga ${ }^{25,27,29}$ e nível de satisfação ${ }^{25}$ de mulheres mastectomizadas.

Sob essa perspectiva, a intervenção Yoga demonstrou ser útil como aditivo no tratamento antiemético em um estudo comparativo em mulheres diagnosticadas com câncer de mama em período de quimioterapia. Os pesquisadores observaram significativa redução dos episódios de náuseas, bem como diminuição na frequência e na severidade das náuseas e vômitos antecipados nas mulheres quando comparadas às submetidas a uma terapia de suporte para o enfrentamento da doença. Além disso, também foi verificada uma pró-influência na diminuição do traço e estado de ansiedade, nos níveis de depressão e nos sintomas de estresse dessas mulheres ${ }^{26}$. 
Durante a radioterapia foram observados benefícios às mulheres portadoras de câncer de mama em um estudo comparando aplicação da intervenção Yoga, durante seis semanas com práticas de 90 minutos, com a terapia de suporte e aconselhamento. Ocorreu neste grupo significativa diminuição nos níveis de ansiedade, depressão e percepção do estresse, enquanto no controle aumentou. Houve também uma diminuição da radiação nociva da radioterapia no DNA das mulheres submetidas a intervenção Yoga quando comparadas ao grupo de terapia ${ }^{6}$.

Partindo do princípio de que a hormonioterapia exacerba os efeitos da menopausa, Carson et al. ${ }^{29}$ observaram benefícios em mulheres sobreviventes de câncer de mama com sintomas de menopausa após oito semanas com $120 \mathrm{minu}$ tos de prática de Yoga, e após três meses de acompanhamento quando comparadas ao controle. Houve melhoria significante na frequência e na severidade das alterações súbitas de temperatura, dores articulares, distúrbios do sono e vigor após as oito semanas. Após os três meses de acompanhamento foram mantidos os ganhos obtidos anteriormente, além de significativos outros no humor negativo, relaxamento e aceitação dessas mulheres.

Diante dos estudos expostos, observa-se uma gama de efeitos gerais e isolados, mensurados nas pacientes mastectomizadas em diferentes fases do tratamento, e em diferentes condições. $\mathrm{O}$ presente estudo, por sua vez, provou que o $\mathrm{Ha}$ tha-Yoga pode trazer benefícios às mulheres mastectomizadas de um modo geral, uma vez que abarcou mulheres em diferentes fases do tratamento. No entanto, fazem-se necessários estudos testando efeitos da intervenção em fases isoladas do tratamento, bem como a comparação dos efeitos nas diferentes fases.

Um outro ponto de questionamento neste estudo foi a falta de controle orgânico dos parâmetros mensurados nas pacientes, ou seja, os níveis de estresse e ansiedade diminuíram baseados nos questionários e sensações relatadas no diário de campo pelas mulheres voluntárias. Neste caso, o período de intervenção deveria ser mais longo como observaram Vadiraja et al. ${ }^{25}$ em um estudo controlado, ao comparar os efeitos da intervenção Yoga durante seis semanas em mulheres mastectomizadas com estádio II e III em radioterapia. Apesar da intervenção ter mostrado diminuição significativa nos níveis de ansiedade, depressão e percepção do estresse e de cortisol salivar, este nível dosado não apresentou redução tão significante quanto os demais que foram relatados pelas voluntárias durante a entrevista. Quanto a isso os autores argumentaram que em pacientes com câncer o estresse diminui normalmente com o tempo, e que esse processo não é uniforme. Uma intervenção como o Yoga acelera esse processo, mas que em pacientes com altos níveis de cortisol iniciais a resposta de diminuição do organismo é mais lenta.

Repossi ${ }^{30}$, em um estudo controlado, ao avaliar os efeitos da intervenção-relaxamento no sistema imunológico de mulheres mastectomizadas após uma semana, não constatou diferença estatística nos níveis de imunoglobulina salivar (molécula ligada à imunidade) ao trabalhar uma única vez a intervenção e ter incentivado a prática domiciliar. Amorim ${ }^{8}$ também utilizou a mesma técnica de relaxamento em mulheres mastectomizadas, desde o diagnóstico até o término do tratamento, e encontrou aumento significativo das células natural killer no grupo experimental.

Ao final de cada intervenção, ao serem questionadas sobre suas sensações, de um modo geral, as mulheres relatavam bem-estar, tranquilidade, relaxamento, paz, leveza e alívio. Essas sensações também foram relatadas no diário de campo no qual as voluntárias relataram os acontecimentos de suas vidas durante o período de intervenção:

...Achei o yoga uma experiência ótima que tive, me sinto mais calma. Tentei fazer algumas coisas em casa quase todos os dias, me fez bem.

... me sinto muito bem, tenho vontade de respirar, respirar, respirar...

... a prática da Ioga tem me mostrado que praticando-a vencemos as limitações que impomos ao nosso corpo.

...Praticar Ioga é como tomar anestesia, deixa o corpo sem dor e sem querer despertá-lo.

...hoje fiz yoga lá no prema. Voltei de lá me sentindo muito bem, cheguei com disposição, não senti desanimo.

As práticas de conexão mente-corpo, de um modo geral, traduzem-se na busca do ser humano por quietude, bem-estar físico e mental, paz e felicidade. Elas têm como objetivo nos fazer tomar contato com o corpo e com a interioridade e funcionam como exercícios de condução mental possibilitando uma mudança radical na forma e no foco dos nossos pensamentos e acarretando transformações em nossas emoções mais frequentes, inclusive no funcionamento dos sistemas orgânicos. Esta compreensão torna-se indispensável para mobilização dos indivíduos a tomar consciência do seu corpo e apropriar-se do processo de cura $^{31}$. 
Assim, o Hatha-Yoga diz respeito a uma aplicação filosófica e prática que leva o praticante a voltar-se ao momento presente, perceber-se física e mentalmente estabelecendo uma conexão corpo-mente, conhecer melhor suas limitações, apreciar-se e respeitar-se. Essa técnica torna-se um poderoso instrumento para o profissional da saúde por enxergar o paciente em sua totalidade bem como fazê-lo "se enxergar", em especial o fisioterapeuta, à medida que este passa a trabalhar o lado psíquico do paciente para reabilitar o seu corpo físico.

\section{Conclusão}

A partir dos resultados considerados, pode-se admitir a intervenção Hatha-Yoga como prática facilmente internalizada pelas mulheres com câncer de mama em curto prazo. O Hatha-Yoga funciona como ferramenta que auxilia a mulher no enfrentamento do câncer de mama à medida que promove o seu autoconhecimento, a melhora de sua autoestima e o gerenciamento de sua ansiedade e estresse, assim como a favorece suas relações externas.

\section{Colaboradores}

MLD Bernardi, MHC Amorim, E Zandonade, DF Santaella e JAN Barbosa participaram igualmente de todas as etapas de elaboração do artigo. 


\section{Referências}

1. Instituto Nacional do Câncer (INCA). Estimativa 2010 - Incidência de câncer no Brasil. Rio de Janeiro: INCA; 2009. [página na Internet]. [acessado 25 set 2013]. Disponível em: http://www.inca.gov.br/ estimativa/2008/

2. Instituto Nacional do Câncer (INCA). Ações de enfermagem para o controle do câncer: uma proposta de integração de serviço. $3^{a}$ Edição. Rio de Janeiro: INCA; 2008.

3. Santos DB, Vieira EM. Imagem corporal de mulheres com câncer de mama: uma revisão de literatura. Cien Saude Colet 2011; 16(5):2511-2522.

4. Simeão SFAP, Landro ICR, Conti MHSD, Gotti MAN, Delgallo WD, Vitta AD. Qualidade de vida em um grupo de mulheres acometidas pelo câncer de mama. Cien Saude Colet 2013; 18(3):779-788.

5. Lipp MEN, Tanganelli MS. Stress e qualidade de vida em magistrados da justiça do trabalho: diferenças entre homens e mulheres. Psicol Reflex Crít 2002; 15(3):537-548.

6. Banerjee B, Vadiraj HS, Ram A, Rao R, Jayapal M, Gopinath KS, Ramesh BS, Rao N, Kumar A, Raghuram N, Hegde S, Nagendra HR, Prakash Hande M. Effects of an integrated yoga program in modulating psychological stress and radiation-induced genotoxic stress in breast cancer patients undergoing radiotherapy. Integr Cancer Ther 2007; 6(3):242-250.

7. Silva $G$, Santos MA. Será que não vai acabar nunca?: perscrutando o universo do pós-tratamento do câncer de mama. Texto Contexto Enferm 2008; 3(17):561-568.

8. Amorim MHC. A enfermagem e a psiconeuroimunologia no câncer da mama [tese]. Rio de janeiro: Universidade Federal do Rio de Janeiro; 1999.

9. López Gonzalez V, Díaz-Páez Waterland A. Efectos del Hatha-Yoga sobre la salud. Parte II. Rev Cuba Med Gen Integr 1998; 14(5):499-503.

10. Gharote ML. Técnicas de Yoga. Guarulhos: Phorte; 2000.

11. Pashard O. Role of yoga in stress management. West Indian Med J 2004; 53(3):191-194.

12. Feuerstein G. A tradição do Yoga: história, literatura, filosofia e prática. 5 Edição. São Paulo: Pensamento; 2006.

13. Danucalov MAD, Simões RS. Neurofisiologia da meditação. São Paulo: Phorte; 2006.

14. Barros NF, Siegel P, Moura SM, Cavalari TA, Silva LG, Furlanetti MR, Gonçalves AV. Yoga e promoção de saúde. Cien Saude Colet 2013. [No prelo].

15. Smith KB, Pukall C. An evidence-based review of Yoga as a complementary intervention for patients with cancer. Psycho-Oncology 2009; 18(5):465-475.

16. Culos-Reed SN, Carson LE, Daroux LM, HateyAldous S. A pilot study of yoga for breast cancer survivors: physical and psychological benefits. Psycho-Oncology 2006; 15(10):891-897.

17. Buettner C, Kroenke CH, Philips RS, Davis RB, Eisenberg DM, Holmes MD. Correlates of use of different types of complementary and alternative medicine by breast cancer survivors in the nurses' health study. Breast Cancer Res Treat 2006; 100(2):219-227.
18. Spielberger CD, Charles D, Gorsuch RL, Lushene R, Vagg PR, Jacobs GA. Manual for the state-traitanxiety inventory. Palo Alto: Consulting Psychological Press; 1970.

19. Spielberg CD, Gorsuch RL, Lushene RE. Inventário de ansiedade traço-estado. Rio de Janeiro: CEPA; 1979.

20. Vasconcelos ED. Stress, coping ans sozial e kompetenz bei kardiovaskularen erkrankugen [tese]. Murchen: Ludwig Maximmlians Universitat; 1984.

21. Brasil. Ministério da Saúde (MS). Conselho Nacional de Saúde. Resolução no 196 de 10 de outubro de 1996. Diretrizes e Normas Regulamentadoras de Pesquisas Envolvendo Seres Humanos. Diário Oficial da União 1996; 16 out.

22. Paraguassú TC, Nogueira TP. Stress Simptoms in Mastectomized Women. VII Conference global Network of WHO Collaborating Centers for Nursing and Midwifery; 28 a 30 jul 2010; São Paulo.

23. Rao R, Raghuram N, Nagendra HR, Gopinath KS, Srinath BS, Diwakar RB, Patil S, Bilimagga SR, Rao N, Varambally S. Anxiolytic effects of o yoga program in early breast câncer patients undergoing conventional treatment: a randomized controlled trial. Complement Ther Med 2009; 17(1):1-8.

24. Ulger O, Yagli NV. Effects of yoga on the quality of life in cancer patients. Complement Ther Med 2010; 16(2):60-63.

25. Vadiraja HS, Raghavendra RM, Nagarathna R, Nagendra HR, Rekha M, Vanitha N, Gopinath KS, Srinath BS, Vishweshwara MS, Madhavi YS, Ajaikumar BS, Ramesh BS, Nalini R, Kumar V. Effects of o yoga programo $\mathrm{n}$ cortisol rhythm and mood states in early breast câncer patients undergoing adjuvant radiotherapy: a randomized controlled trial. Integr Cancer Ther 2009; 8(1):37-45.

26. Raghavendra RM, Nagarathna RM, Nagendra HR, Gopinath KS, Srinath BS, Ravi BD, Patil S, Ramesh BS, Nalini R. Effects of an integrated yoga programme on chemotherapy-induced nausea and emesis in breast cancer patients. Eur J Cancer Care 2007; 16(6):462-474.

27. Moadel AB, Shah C, Wylie-Rosett J, Harris MS, Patel SR, Hall CB, Sparano JA. Randomized controlled trial of yoga among a multiethnic sample of breast cancer patients: effects on quality of life. $J$ Clin Oncol 2007; 25(28):4387-4394.

28. Bower JE, Garet D, Sternlieb B. Yoga for Persistent Fatigue in Breast Cancer Survivors: Results of a Pilot Study. Evid Based Complement Alternat Med 2011; 2011:623168.

29. Carson JW, Carson KM, Porter LS, Keefe FJ, Seewaldt VL. Yoga of awareness program for menopausal symptoms in breast cancer survivors: results from a randomized trial. Support Care Cancer 2009; 17(10):1301-1309.

30. Repossi C. Efeitos da intervenção enfermagem-relaxamento no sistema imunológico de mulheres mastectomizadas [dissertação]. Vitória: Universidade Federal do Espírito Santo; 2008. 
31. Alminhana LO. Benefícios das técnicas mente-corpo como intervenções psicoterapêuticas em pacientes oncológicos em tratamento quimioterápico, sob a perspectiva transpessoal [dissertação]. São Leopoldo: Instituto Ecumênico de Pós Graduação da Escola Superior de Teologia; 2004.

Artigo apresentado em 16/08/2012

Aprovado em 03/10/2012

Versão final apresentada em 08/10/2012 